\title{
CAN VORONOI DIAGRAM MODEL CELL GEOMETRIES IN EARLY SEA-URCHIN EMBRYOGENESIS?
}

\author{
M.A.Luengo-Oroz $z^{\S}$, L.Duloquin ${ }^{\natural}$, C.Castro ${ }^{\S}$, T.Savy ${ }^{\ddagger}$, E.Faure ${ }^{\ddagger}$, B.Lombardot ${ }^{\ddagger}$, \\ P.Bourgine ${ }^{\ddagger}$, N.Peyriéras $s^{\natural}$ and A. Santos ${ }^{\S}$
${ }^{\S}$ Biomedical Image Technologies lab., ETSI Telecomunicación, Universidad Politécnica de Madrid, Spain ${ }^{\ddagger}$ Centre de Recherche en Epistémologie Appliquée, CNRS - École Polytechnique, Paris, France ${ }^{\natural}$ DEPSN, CNRS - Institut de Neurobiologie Alfred Fessard, Gif-sur-Yvette, France

\begin{abstract}
We test the hypothesis that cell membranes in early seaurchin embryos can be modeled as a Voronoi diagram from nuclei centers. In order to obtain a model of the cell geonetry against which to test our Voronoi model hypothesis, we developed a viscous watershed framework that allows segmenting 3D images of living sea-urchin embryos obtained by biphoton laser scanning microscopy. Measurements of the differences between segmented cells and the Voronoi model, show an interesting high correlation that can serve for developing more accurate methods of segmentation and modelling cell geometries.
\end{abstract}

Index Terms- Microscopy, morphological operations, biological cells, image segmentation.

\section{INTRODUCTION}

Reconstructing cell morphologies throughout embryonic development in live animals is of great interest for bio-medical research[1]In this work, we try to fill the gap between computer vision segmentation methods and mathematical models of cell geometries with measurements in real experiments. We test the hypothesis that cell membranes in early sea-urchin embryogenesis can be modeled as a Voronoi diagram from nuclei centers detected in the live animal imaged by biphoton laser scanning microscopy. This kind of hypothesis has been previously used to simulate tissue biomechanics [2][3], but no real analysis of its accuracy has been done. For that purpose, we have created a golden standard of cell membranes using morphological segmentation methods. This segmentation is compared to the synthetic membranes created with Voronoi diagrams starting from real detected nuclei. Measurements on these comparisons, show how accurate is the model and can be used to develop more complex theoretical models of cell biomechanics. This paper is organized as follows(see Fig. 1): in section 2 we present a brief description of sea-urchin embryogenesis and the imaging procedure. In section 3 , image processing methods for cell segmentation are presented and in section 4 , we describe the construction of Voronoi membranes. Finally, we analyze the results of the comparison of both methods and discuss them.

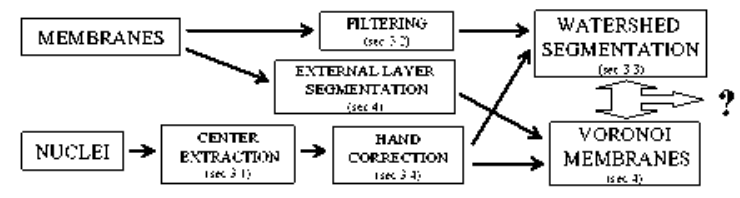

Fig. 1. Overview of the processing methods.

\section{IMAGING SEA-URCHIN EMBRYOGENESIS}

Sea urchin embryo is a useful animal model system because of its phylogenetic position (deuterostomes, echinoderm), its rapid development and the complete transparency of its embryo that allows microscopic observation. Sea urchins of the species Paracentrotus lividus have an embryonic development that can be divided in two main steps: the clivage period, which goes on for the first 10 hours of development, and the gastrulation allowing the formation of the digestive tract and skeleton. Development leads to a larva composed of about 1500 cells at 36 hours post fertilization(hpf). In the experiment exploited in this paper, we have imaged the whole living embryo at $15 \mathrm{hpf}$ i.e. during the gastrulation period. This $3 D$ dataset is obtained through time-lapse laser scanning nicroscopy from live embryos engineered to fluorescently[4] label nuclei and membranes (see Appendix for details). At $15 \mathrm{hpf}$, embryo has about 850 cells (see Fig. 2). It looks like in a empty ball with an outside layer of tightly joint epithelial cells. In the central cavity, 32 cells have ingressed and are spatially organized. Ingression and migration of these cells are two main morphogenetic events that have been extensively studied. This process is similar to the migration of metastatic cells during cancer progression. Soon after this 
cell ingression step, the digestive tract forms by invagination in the same region. The genetic networks implicated in such events begin to be well described and research in next years should fill gaps between genetic and cellular behaviors.

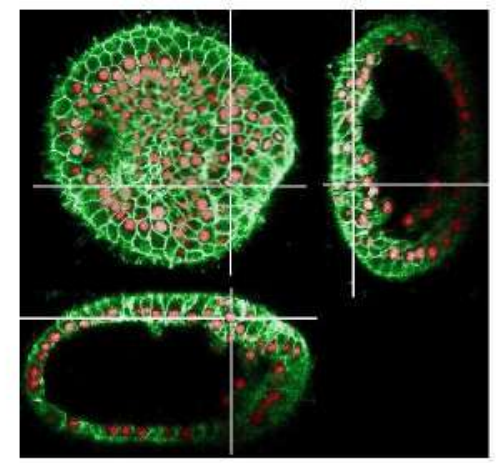

Fig. 2. Cross-sections of the imaged dataset(15hpf). Membranes in green and nuclei in red.

\section{MORPHOLOGICAL CELL MEMBRANE SEGMENTATION}

Segmentation is achieved through the classical marker based watershed paradigm (see algorithms parameters in Appendix): the filtered membrane image is the mask image and the nuclei image is used to obtain the marker image. Viscous flooding is used for the labeling process.

\subsection{Nuclei extraction}

Nuclei detection is the most delicate stage in watershed based segmentation[5]. Since our objective is to segment and to build the Voronoi diagram from a perfect nuclei detection, we will hand correct the automatic detection. For this reason, we have chosen a simple morphological method that produces easily manually correctible errors: it provides no false positives and errors just coming from two merged nuclei. Nuclei image is preprocessed with a 3D median filter and smoothed with a small spherical gaussian kernel. Afterwards, a smoothed version of the corresponding membranes image is subtracted to the nuclei image in order to remark the separation between merged nuclei(a membrane is placed between two nuclei in most of the cases). Then, the extraction is achieved by means of residues of area openings (in the sense of greyscale area opening of [6] extended to 3D). Let $\chi_{h}(f)$ be the threshold function for the greylevel $h$ and let $f$ be the input image, let $\gamma_{B}^{v}$ be the greyscale area opening for a value $v$ and a ball structuring element $B$; so $\gamma_{B}^{v}(f(x))=\sup \left\{h \leq f(x) \mid\right.$ Area $\left.\left(\gamma_{B}\left(\chi_{h}(f)\right)\right) \geq v\right\}$. The reconstructed nuclei image $N$ is given by the objects whose volume is in $\left[v_{1}, v_{2}\right]$. The values $v_{1}, v_{2}$ are chosen empirically. Finally, nuclei centers are identified as regional maxima, so $N=\operatorname{Reg} \operatorname{Max}\left(\gamma_{B}^{v_{1}}(f)-\gamma_{B}^{v_{2}}(f)\right)$.

\subsection{Membrane processing}

Membranes are pre-processed with a $3 \mathrm{D}$ median filter. Secondly, image is filtered with a 3D twister segment morphological filtering[7]. The opening by a twister segment structuring element is the supremum of several morphological opening by different rotations of a $1 \mathrm{D}$ segment in a $\mathrm{nD}$ space. Finally, for regularization purposes, image is convoluted with a $3 \mathrm{D}$ spherical gaussian kernel.

\subsection{Viscous watershed flooding}

Watershed with markers was introduced by [8] and has been extensively used in cell segmentation applications. A well known drawback of watershed methodology is leakage in some poorly defined or fuzzy membrane regions, especially at low intensity levels. Recently, several solutions to the leaking problems have been proposed by introducing some constraints and regularization terms in the region growing procedure[9]. Such an interesting simple alternative consists in flooding the image with viscous liquid that can produce smoother contours and more regular shapes than the standard watershed[10]. An equivalent method to the viscous liquid flooding consists in properly modifying the original topology of the image by means of viscous closings and then performing the standard, non-viscous watershed on it. The oil-model fluid has been chosen for the viscous closing transformation (see Fig. 3) because the cell membrane image has well defined polygonal boundaries with high intensity level and blurred contours with low intensity. In this model temperature is associated to the intensity level $h$. Oil at low temperatures(dark pixels) has high viscosity and frontiers between regions are smoothed because fluid does not leak. At high altitude, with high temperature, oil is less viscous and watershed lines carefully follow relief like standard watershed lines do. The viscous closing transformation $T(f)$ may be implemented by adding the binary closings of image $f$ at all the intensity levels $h>0$ with a ball of radius $r(h)$, $T(f)=\vee_{h \geq 0} \varphi_{r(h)}\left(h \cdot \chi_{h}(f)\right)$ with $\chi_{h}(f)(p)=1$ for all $p \in \chi_{h}(f)$ and $\chi_{h}(f)(p)=0$ elsewhere. Notice $h \cdot \chi_{h}(f)$ corresponds to the level set $\chi_{h}(f)$ at the altitude $h$. The function $r(h)$ defines the behaviour of the fluid at different levels. oil-model function $r(h)=R_{\max }-h * R_{\text {step }}$ performed for $r(h)>0$. The choice of $R_{\max }$ is directly related to cell size (if $r(h)$ is too big, details of cell shape are lost) and $R_{\text {step }}$ is related to the level $h_{v}=R_{\max } / R_{\text {step }}$ from where viscosity effects are neglected.

\subsection{Segmentation method validation}

We have manually validated our detection method using InsigthSNAP software[11]. The whole dataset was validated in 6 hours by an expert eye. The analyzed dataset has 827 nuclei. Our method has correctly identified 613 nuclei (74\%). In 52 


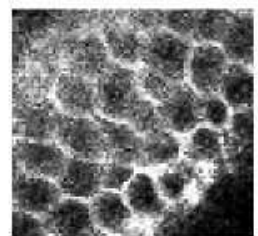

(a)

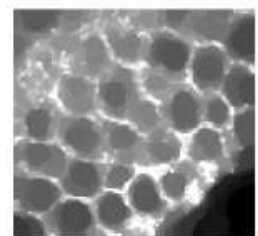

(b)



(c)
Fig. 3. (a) Membranes image; (b) Viscous closing of (a); (c) Comparison of standard (red) and viscous (green) watershed.

cases, the algorithm has identified one nuclei when they were actually two merged (104 nuclei, $12.5 \%$ ). The remaining 110 nuclei $(13.5 \%)$ have been manually added, providing the nuclei model that is used in further stages of segmentation and Voronoi reconstruction. Membranes segmentation has been evaluated by visual inspection, assigning whether good or bad segmentation was done. The percentage of good segmented cells decreases with depth, as signal looses quality. Because of that we have evaluated the segmentation at $10,20,40$ and $60 \mu \mathrm{m}$ from the top of the embryo (total embryo diameter is about $75 \mu \mathrm{m}$ ). Respectively $78 \%, 65 \%, 43 \%$ and $45 \%$ of cells were well segmented.

\section{SYNTHETIC MEMBRANES RECONSTRUCTION WITH VORONOI DIAGRAM}

In order to reconstruct the synthetic membranes, we built the Voronoi diagram (see Fig. 4) from the manually-corrected nuclei $E$ extracted in Section 3.4 and bounded it to the external embryo layer $T$. This layer defines the region where cell membranes are confined and it is extracted by taking the morphological gradient of the threshold of the original membrane image. The value of the threshold function was found manually and the result was regularized with a spherical closing. The Voronoi diagram[12] of a set $E=\cup e_{i}$ of seeds in a space $T$ provided with the Euclidean metric is the union of boundaries of the Voronoi regions associated to each particle $e_{i}$. Each Voronoi region, $V R\left(e_{i}\right)$, is composed by the points of $T$ which are not closer to other point $e_{j}$ than to $e_{i}$ (in morphological terms Skeleton of Influence Zones).

\section{RESULTS}

The similarity between segmented cell membranes and the modeled Voronoi membranes has been quantitatively assessed in 100 manually selected cells, comparing the correctly segmented membranes with the Voronoi regions associated to their nuclei center. Our similarity metric $\Phi$ is given by the value $\Phi($ seg, vor $)=\sharp($ seg $\cap$ vor $) / \sharp($ seg $)$, which represents the percentage of the segmented cell which is covered by its corresponding Voronoi region. Results are shown in Fig. 5, the mean value is 0.71 , the median 0.73 and standard deviation 0.24 . There are the same number of nuclei centers and

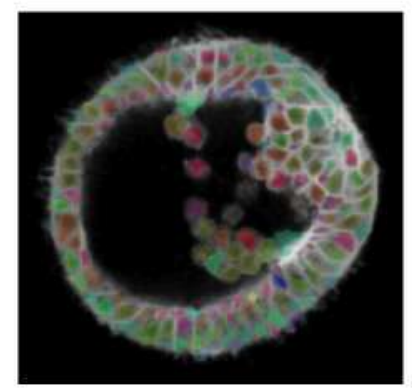

(a)

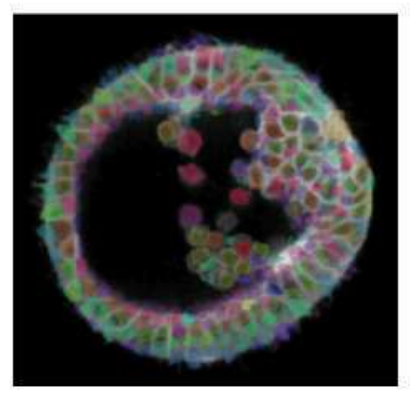

(b)
Fig. 4. (a) Segmented data and (b) Voronoi regions superimposed to the original membranes image.

the total volume is the same for segmented data and Voronoi regions. Notice that these statistics are biased from real data because of non absolutely perfect segmentations.
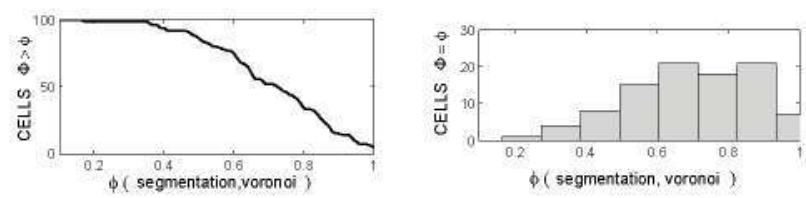

Fig. 5. Measurements on similarity $\Phi$ between cell segmentation and its corresponding associated Voronoi model. Left: number of cells with higher similarity $\Phi$ than a given value $\phi$, Right: histogram of number of cells with similarity values $\Phi \in[\phi-0.05, \phi+0.05], \phi=\{0.05,0.15,0.25, \ldots\}$.

\section{DISCUSSION AND CONCLUSIONS}

This work has several implications that can serve both image processing and biological modelling. We have designed and validated a semi-automatic watershed based methodology that allowed us to segment cell membranes in 3D image data sets from live early sea urchin embryos. This methodology has been used to obtain a representation of cell geometries that has been compared with the Voronoi model of cell membranes. The high correlation showed in this comparison justifies the use of Voronoi model in literature[2][3] and its potential value for image segmentation. Using Voronoi membranes can be interesting in order to design a synthetic embryo, particularly when low signal to noise ratio produces poorly defined membranes and causes failure in traditional segmentation paradigms. In addition, substituting the Euclidean metric used in the Voronoi construction by another metric dependent on the image contents [13] could serve as a basis for a refined model. Finally, the Voronoi presented here could serve as a precise starting point for segmentation methods such as snakes. Qualitative biological observations allow us to formulate the new hypothesis that similarity between Voronoi 
membranes and cell geometries is a significant feature that should fit with specific biomechanical properties of the tissue and underlying molecular and genetic network properties. We interpret the matching between Voronoi diagram and cell geometries as and indication of the pressure encountered by the cells in their morphogenetic field. In other words, Voronoi models would profile cells arrangement whenever they act as passive structures. The cell wall of the early sea urchin embryo investigated in this paper provides the example of an epithelium with probably a good equilibrium between the cells and little source of symmetry breaking such as cell division or cell intrinsic movements. Conversely, any rupture in this equilibrium correlating with molecular and genetic changes should turn into detectable differences between actual membranes geometry and Voronoi diagram. We propose this criterion as a tool for investigating morphogenetic fields characteristic features and future work is open to make more detailed comparisons at different scales and development stages.

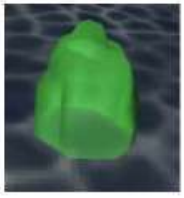

(a)



(b)

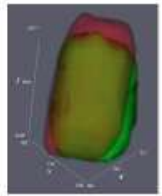

(c)
Fig. 6. (a) Cell segmentation, (b) Voronoi model, (c) Similarity $\Phi(a, b)=0.77$.

\section{Appendix}

\section{Imaging parameters}

Nuclei and cellular membranes were labellled with fluorescent proteins. mRNAs encoding $\mathrm{H} 2 \mathrm{~B}$-mCherry(nuclei) and eGFP-Ras(membranes) proteins were injected into the egg at the one cell stage. Imaging has been done with a biphoton laser scanning microscope (Leica SP5) equipped with a Leica objective HCX PL APO CS 63x/1.20NA W CORR. The embryo was maintained between a slide and a cover slide, both were previously coated with $2 \%$ protamine sulfate. Image size is $512 \times 512 \times 86$ pixels and voxel size is $(0.48 \times 0.48 \times 0.96) \mu m^{3}$. Acquisition time is 57.1 seconds.

\section{Algorithms parameters}

Size and radius are expressed in pixels.

NUCLEI EXTRACTION (SEC.3.1): MED( $3 \times 3 \times 3)$, GAUS(5), AREA OPENING $\left(v_{1}=150 ; v_{2}=600\right)$; MEMBRANE PROC (SEC.3.2): MED(3X3X3), TWISTER(7), GAUS(3); VISCOUS FLOODING(SEC.3.3): VISCOUS CLOSING $\left(R_{\max }=5, R_{\text {step }}=\right.$ 0.1 ); EXTERNAL LAYER (SEC.4): THRESHOLD(25), GAUS(5).

\section{REFERENCES}

[1] S.G.Megason and S.E.Fraser, "Digitizing life at the level of the cell: high-performance laser-scanning microscopy and image analysis for in toto imaging of development," Mech.Dev., vol. 120, pp. 1407-1420, 2003.

[2] H. Honda, M. Tanemura, and T. Nagai, "A threedimensional vertex dynamics cell model of space-filling polyhedra simulating cell behavior in a cell aggregate," J. Theor. Biol, vol. 226, no. 4, pp. 439-53, 2004.

[3] M. Weliky, "The mechanical basis of cell rearrangement. I, Epithelial morphogenesis during Fundulus epiboly," Development, vol. 109, no. 2, pp. 373-386, 1990.

[4] D.M. Chudakov, S. Lukyanov, and K.A. Lukyanov, "Fluorescent proteins as a toolkit for in vivo imaging.," Trends Biotechnol., vol. 23, pp. 605-13, Dec 2005.

[5] N. Malpica, C.O. de Solorzano, J.J. Vaquero, A. Santos, I. Vallcorba, J.M. Garcia-Sagredo, and F. del Pozo, "Applying watershed algorithms to the segmentation of clustered nuclei," Cytometry, vol. 28, no. 4, pp. 289297, 1997.

[6] L. Vincent, "Morphological area opening and closing for grayscale images," in Shape in Picture Workshop. NATO, Springer-Verlag, 1992, pp. 197-208.

[7] M.A. Luengo-Oroz, E. Faure, B. Lombardot, R. Sance, P. Bourgine, N. Peyrieras, and A. Santos, "Twister segment morphological filtering. a new method for live zebrafish embryos confocal images processing," in IEEE ICIP, San Antonio, Texas, 2007, pp. 253-256.

[8] F. Meyer and S. Beucher, "Morphological segmentation," J.Vis.Commun.Image Represent., vol. 1, pp. 2146, Sep 1990.

[9] R. Beare, "A locally constrained watershed transform," IEEE Trans. Pattern Anal. Mach. Intell., vol. 28, no. 7, pp. 1063-1074, 2006.

[10] C.Vachier and F.Meyer, "The viscous watershed transform," J. Math. Imaging Vis., vol. 22, no. 2-3, pp. 251$267,2005$.

[11] G. Gerig, P. Yushkevich, and S. Ho, "ITK-SNAP," www. itksnap.org.

[12] R. Fabbri, L.F. Estrozi, and L. da Fontoura Costa, "On voronoi diagrams and medial axes," J. Math. Imaging Vis, vol. 17, no. 1, pp. 27-40, July 2002.

[13] T.R. Jones, A. Carpenter, and P. Golland, "Voronoibased segmentation of cells on image manifolds," CVBIA05, LNCS, vol. 3765, pp. 535-543, 2005. 